\title{
Joint reconstruction framework of compressed sensing and nonlinear parallel imaging for dynamic cardiac magnetic resonance imaging
}

\author{
Zhanqi Hu ${ }^{1 \dagger}$, Cailei Zhao ${ }^{1 \dagger}$, Xia Zhao ${ }^{1}$, Lingyu Kong ${ }^{1}$, Jun Yang ${ }^{2}$, Xiaoyan Wang ${ }^{3^{*}}$, Jianxiang Liao ${ }^{{ }^{*}}$ and \\ Yihang Zhou ${ }^{4^{*}}$
}

\begin{abstract}
Compressed Sensing (CS) and parallel imaging are two promising techniques that accelerate the MRI acquisition process. Combining these two techniques is of great interest due to the complementary information used in each. In this study, we proposed a novel reconstruction framework that effectively combined compressed sensing and nonlinear parallel imaging technique for dynamic cardiac imaging. Specifically, the proposed method decouples the reconstruction process into two sequential steps: In the first step, a series of aliased dynamic images were reconstructed from the highly undersampled k-space data using compressed sensing; In the second step, nonlinear parallel imaging technique, i.e. nonlinear GRAPPA, was utilized to reconstruct the original dynamic images from the reconstructed k-space data obtained from the first step. In addition, we also proposed a tailored k-space down-sampling scheme that satisfies both the incoherent undersampling requirement for $\mathrm{CS}$ and the structured undersampling requirement for nonlinear parallel imaging. The proposed method was validated using four in vivo experiments of dynamic cardiac cine MRI with retrospective undersampling. Experimental results showed that the proposed method is superior at reducing aliasing artifacts and preserving the spatial details and temporal variations, compared with the competing $k$-t FOCUSS and k-t FOCUSS with sensitivity encoding methods, with the same numbers of measurements.
\end{abstract}

Keywords: Dynamic cardiac MRI, Compressed sensing, Parallel imaging, Nonlinear GRAPPA

\section{Introduction}

Dynamic Magnetic resonance imaging (dMRI) is an important medical imaging modality for the diagnosis of cardiovascular diseases. Due to its excellent tissue

\footnotetext{
*Correspondence: wxy@yxnu.edu.cn; liaojianxiang@vip.sina.com; yihang zhou@outlook.com

${ }^{\dagger}$ Co-first author: Zhanqi Hu and Cailei Zhao

1 Shenzhen Children's Hospital, Shenzhen 518038, Guangdong, China

${ }^{3}$ School of Physics and Electronic Engineering, Yuxi Normal University, Yuxi 653100, Yunnan, China

${ }^{4}$ Hong Kong Sanatorium and Hospital, 5 A Kung Ngam Village Road, Shau Kei Wan, Hong Kong, China

Full list of author information is available at the end of the article
}

contrast, MRI can effectively evaluate cardiac functions and vascular abnormalities by acquiring a time series of images at a high frame rate during cardiac motion. To obtain an artifact-free image series using conventional Fourier reconstruction, the Nyquist sampling requirement must be satisfied in both spatial and temporal directions. However, due to the low data acquisition speed, dynamic MRI does not always meet this criterion. Hence, dynamic MRI often suffers from aliasing or motion artifacts. The acquisition speed is hence of primary importance for achieving high spatial-temporal resolution in dynamic MRI applications. A range of techniques have been developed to reconstruct a high-quality image series 
from the undersampled MRI data by exploiting spatial and/or temporal correlations in the dynamic image series. These spatial and temporal correlations render it feasible to estimate the missing data from undersampled measurements. Typical methods for the reconstruction of undersampled single-coil measurements include RIGR [1], keyhole [2], view-sharing [3], UNFOLD [4], Partially Separable Function (PS) [5-7], Kalman filter [8], PARADIGM [9], k-t BLAST [10] and so on.

Two promising techniques for speeding up acquisition are parallel imaging and compressed sensing, which downsample the k-space below the Nyquist rate. Parallel imaging [11-14] uses information from multiple coil signals to estimate the unacquired $\mathrm{k}$-space data, such as the classical SENSE [12] and GRAPPA [13]. Theoretically, the maximum acceleration rate of parallel imaging can be up to the number of physical channels under ideal conditions. However, noise and imperfect coil geometry limit the achievement to the maximum acceleration in practice. Compressed sensing (CS) [15-17] exploits the sparseness of the signal in a certain domain as the prior constraint and recovers the accurate signal using nonlinear optimization algorithms. The success of applying CS to dynamic cardiac MRI greatly accelerates the acquisition process [18-26]. Such success is based on two important properties of the dynamic cardiac images: firstly, the dynamic cardiac images exhibit strong correlations between frames which guarantee the sparse representation of the sequence in a specific transform domain; secondly, the sampling pattern can be easily designed to satisfy the incoherence requirement of CS theory.

Since the sampling scheme of CS and parallel imaging exploit complementary redundancy information in MRI, it is of great interest to combine these two techniques so that a higher reduction factor could be achieved. Several studies attempted to combine parallel MRI (pMRI) and CS for static imaging [27-39]. Conventionally, the multicoil information was incorporated into the CS framework by replacing the Fourier encoding with sensitivity encoding in the data consistency term [25, 27, 35], together with any sparsifying transform such as the low-rank model [31, 32], the dictionary learning [33, 34]. In addition, the recent development of deep learning techniques also attempted to combine the CS and pMRI frameworks into various cardiac MRI applications [37-39]. However, few studies explored how to efficiently combine CS and pMRI approaches to maximize accelerations, given the different sample requirements of the two approaches.

In this paper, we proposed a novel reconstruction framework that efficiently combines compressed sensing and non-linear parallel imaging technique to accelerate dynamic cardiac imaging, extending results in our conference papers [40, 41]. The proposed framework decouples the reconstruction process into two sequential steps. In the first step, the highly undersampled k-space data is used to recreate a series of aliased dynamic cardiac images using a CS approach; In the second step, nonlinear GRAPPA [42-44] was used to remove the aliasing artifacts and recover the desired dynamic cardiac cine images. In addition, the sampling pattern for each step is also designed accordingly, allowing for simultaneous satisfaction of the incoherent undersampling need for CS and the structured undersampling requirement for parallel imaging. The proposed method was validated on four in vivo human cardiac cine MR datasets. Experimental results indicated that the proposed joint reconstruction framework could effectively combine the CS and parallel imaging to improve the reconstruction quality of dynamic cardiac MRI, comparing to the conventional methods.

\section{Theory and method}

In the data acquisition process, the $\mathrm{k}$ - $\mathrm{t}$ space is undersampled by taking a random subset of the already uniformly undersampled k-space data at each time point. Mathematically, let $\mathbf{M}_{\mathrm{u}}$ represent the uniform undersampling operation, $\mathbf{M}_{\mathrm{r}}$ represents the operation of taking a random subset in $\mathrm{k}$-space. The data acquisition process can be expressed by:

$$
\mathbf{d}_{\mathrm{u}, \mathrm{r}}=\mathbf{M}_{\mathrm{r}} \mathbf{M}_{\mathrm{u}} \mathbf{d}_{\text {full }}
$$

where $\mathbf{d}_{\mathrm{u}, \mathrm{r}}$ is the acquired data, and $\mathbf{d}_{\text {full }}$ is the fully sampled reference data in $\mathrm{k}-\mathrm{t}$ space. The uniform undersampling has a reduction factor of $R_{1}$, and the random subset has a reduction factor of $R_{2}$ for the CS requirement. As a result, the total acceleration is $R_{1} \times R_{2}$. For all time frames, additional auto calibration signals (ACS) are also acquired at the center k-space.

Based on the decoupled undersampling operations $\mathbf{M}_{\mathrm{u}}$ and $\mathbf{M}_{\mathrm{r}}$, the reconstruction is carried out in two sequential steps. The CS reconstruction framework is utilized in the first stage, with the goal of reconstructing a uniformly undersampled k-space by "inverting" the operation $\mathbf{M}_{\mathrm{r}}$. Let the aliased image series to be reconstructed in the $(\mathrm{x}-\mathrm{f})$ domain is represented by $\boldsymbol{\rho}_{\mathrm{u}}$. The data consistency term is then given by

$$
\mathbf{d}_{\mathrm{u}, \mathrm{r}}=\mathbf{F} \boldsymbol{\rho}_{\mathrm{u}}
$$

where $\mathbf{F}$ is the Fourier transform along both $x$ and $f$ directions. The reconstruction of the signal $\rho_{\mathrm{u}}$ in $x-f$ domain can be modeled as a truncated $\ell_{1}$ minimization problem

$$
\min _{\boldsymbol{\rho}_{\mathrm{u}}}\left\|\boldsymbol{\rho}_{\mathrm{u}}\right\|_{1} \quad \text { s.t. } \quad\left\|\mathbf{d}_{\mathrm{u}, \mathrm{r}}-\mathbf{F} \boldsymbol{\rho}_{\mathrm{u}}\right\|_{2} \leq \varepsilon
$$

Here we use the k-t FOCUSS [21] algorithm to reconstruct the aliased image sequence in the 
temporal-frequency $(x-f)$ domain, although other methods are also applicable.

The solution to Eq. (3) is computed by iteratively solving a reweighted $\ell 2$ minimization problem defined as

$$
\text { find } \rho_{\mathbf{u}}=\mathbf{W} \mathbf{q}
$$

such that $\mathbf{q}$ is the solution to

$$
\min _{\mathbf{q}}\|\mathbf{q}\|_{2} \quad \text { s.t. } \quad\left\|\mathbf{d}_{\mathrm{u}, \mathrm{r}}-\mathbf{F W q}\right\|_{2} \leq \varepsilon
$$

where $\mathbf{W}$ is a diagonal weighting matrix that is updated iteratively. Equation (4) can be converted into an unconstrained optimization problem by introducing the Lagrangian multiplier $\lambda$,

$$
\min _{\mathbf{q}}\left\|\mathbf{d}_{\mathrm{u}, \mathrm{r}}-\mathbf{F W} \mathbf{q}\right\|_{2}^{2}+\lambda\|\mathbf{q}\|_{2}^{2}
$$

which has a closed-form solution

$$
\mathbf{q}=\mathbf{W}^{\mathbf{H}} \mathbf{F}^{\mathbf{H}}\left(\mathbf{F W} \mathbf{W}^{\mathbf{H}} \mathbf{F}^{\mathbf{H}}+\lambda \mathbf{I}\right)^{-1} \mathbf{d}
$$

Conjugate gradient is used to solve Eq. (6) to avoid the large matrix inversion. So the $x-f$ image is given by

$$
\boldsymbol{\rho}_{\mathrm{u}}=\mathbf{W} \mathbf{q}=\mathbf{W} \mathbf{W}^{\mathbf{H}} \mathbf{F}^{\mathbf{H}}\left(\mathbf{F W} \mathbf{W}^{\mathbf{H}} \mathbf{F}^{\mathbf{H}}+\lambda \mathbf{I}\right)^{-1} \mathbf{d}
$$

Specifically, in the $l$-th iteration, the diagonal elements of the matrix $\mathbf{W}^{(l)}$ are the square root of the absolute value of the solution $\rho_{\mathrm{u}}^{(l-1)}$ from the previous iteration, where $\boldsymbol{\rho}_{n}^{(l-1)}$ is the nth element of $\boldsymbol{\rho}_{\mathrm{u}}^{(l-1)}$. The reconstructed $\boldsymbol{\rho}_{\mathrm{u}}$ is then Fourier transformed along both spatial and temporal frequency directions to obtain the uniformly undersampled data in $k-t$ space.

The missing $\mathrm{k}$-space lines in the reconstructed uniformly undersampled data in $\mathrm{k}-\mathrm{t}$ space from the CS step are then further reconstructed using parallel imaging techniques. To avoid the computation of sensitivity maps and to minimize the error/noise amplification, here we employed nonlinear GRAPPA technique [42]. However, it should be noted that any advanced parallel imaging methods could also be easily integrated into the proposed framework. The pre-acquired ACS lines are used to normalize the reconstructed k-space to correct any mismatch. Specifically, we assume the reconstructed and the acquired data differ by a scaling complex constant:

$$
\mathbf{d}_{\text {recon }}=\beta \mathbf{d}_{\mathrm{acq}}
$$

where $\beta$ is the scaling factor. The $k$-space for each frame is then scaled based on the overlapping locations of reconstructed and acquired data.

Nonlinear GRAPPA [42-44] is conducted after normalization. In nonlinear GRAPPA, the missing data is represented by a nonlinear combination of the acquired data. Here we apply a truncated second-order polynomial whose efficiency and effectiveness have been demonstrated in [42-44]. The missing k-space signal $S_{j}$ in $\mathbf{d}_{\text {recon }}$ is obtained by

$$
\begin{aligned}
S_{j} & \left(k_{y}+r \Delta k_{y}, k_{x}\right)=w_{j, r}^{(0)} \times 1 \\
+ & \sum_{l=1}^{L} \sum_{b=B_{1}}^{B_{2}} \sum_{h=H_{1}}^{H_{2}} w_{j, r}^{(1)}(l, b, h) \times S_{l}\left(k_{y}+b R \Delta k_{y}, k_{x}+h \Delta k_{x}\right) \\
+ & \sum_{l=1}^{L} \sum_{b=B_{1}}^{B_{2}} \sum_{h=H_{1}}^{H_{2}} w_{j, r}^{(2,0)}(l, b, h) \times S_{l}^{2}\left(k_{y}+b R \Delta k_{y}, k_{x}+h \Delta k_{x}\right) \\
+ & \sum_{l=1}^{L} \sum_{b=B_{1}}^{B_{2}} \sum_{h=H_{1}}^{H_{2}-1} w_{j, r}^{(2,1)} \times S_{l}(l, b, h) \times S_{l}\left(k_{y}+b R \Delta k_{y}, k_{x}+(h+1) \Delta k_{y}, k_{x}+h \Delta k_{x}\right) \\
+ & \sum_{l=1}^{L} \sum_{b=B_{1}}^{B_{2}} \sum_{h=H_{1}}^{H_{2}-2} w_{j, r}^{(2,2)}(l, b, h) \times S_{l}\left(k_{y}+b R \Delta k_{y}, k_{x}+h \Delta k_{x}\right)
\end{aligned}
$$

$$
\mathbf{W}^{(l)}=\left(\begin{array}{cccc}
\left|\boldsymbol{\rho}_{1}^{(l-1)}\right|^{0.5} & 0 & \cdots & 0 \\
0 & \left|\boldsymbol{\rho}_{2}^{(l-1)}\right|^{0.5} & \cdots & 0 \\
\vdots & \vdots & \ddots & \vdots \\
0 & 0 & \cdots & \left|\boldsymbol{\rho}_{n}^{(l-1)}\right|^{0.5}
\end{array}\right) \text {, }
$$

where $S_{l}$ represents the acquired k-space data, $w$ is the coefficient set, $R$ is the outer reduction factor, $j$ is the target coil, $l$ counts all coils, $b$ and $h$ transverse the acquired neighboring $\mathrm{k}$-space data in $k_{y}$ and $k_{x}$ directions respectively, and $k_{x}$ and $k_{y}$ represent the coordinates along the frequency- and phase-encoding directions, respectively. The final image is obtained by combining images from 


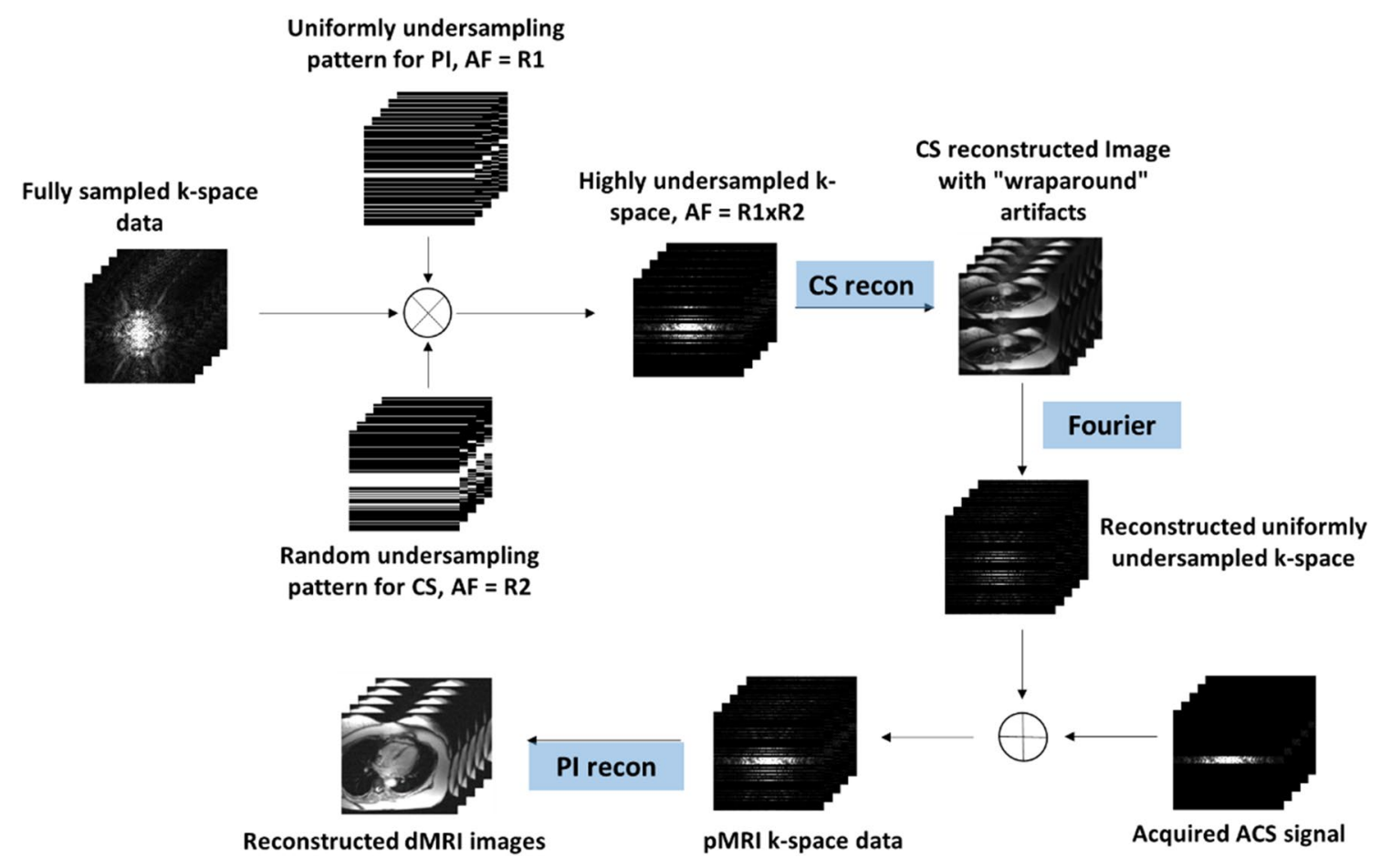

Fig. 1 Reconstruction flowchart of the proposed method

Table 1 Data acquisition parameters for imaging experiments

\begin{tabular}{lllll}
\hline & Data 1 & Data 2 & Data 3 & Data 4 \\
\hline Scanner & 3T Siemens & 3T Siemens & 3T Siemens & 3T Siemens \\
Sequence & SSFP & 2D true FISP & SSFP & SSFP \\
Flip angle & 44 & 50 & 50 & 44 \\
$\begin{array}{l}\text { Echo time } / \text { repetition } \\
\text { time }(\mathrm{ms})\end{array}$ & $1.5 / 3.0$ & $1.87 / 29.9$ & $1.89 / 56.6$ & $1.22 / 42.5$ \\
$\begin{array}{l}\text { Matrix size (FE } \times \text { PE } \times \\
\text { Frame } \times \text { Coil) }\end{array}$ & $166 \times 130 \times 15 \times 5$ & $256 \times 216 \times 20 \times 4$ & $256 \times 224 \times 14 \times 4$ & $304 \times 165 \times 26 \times 12$ \\
\hline
\end{tabular}

all coils using root of sum-of-squares after the missing $\mathrm{k}$-space data for all coils has been recovered. The proposed framework is demonstrated in Fig. 1.

Validation of the proposed method was performed on four sets of cardiac cine data from dynamic MRI, each covering a complete cardiac cycle. The imaging parameters were shown in Table 1. Informed consent was obtained from all volunteers in accordance with the institutional review board policy. The fully sampled data were uniformly undersampled with a reduction factor of $R_{1}$ and furthermore randomly undersampled with a reduction factor of $R_{2}$ using a zero-mean random Gaussian distribution whose density tapers off toward the outer $k$-space retrospectively.
The proposed method, k-t FOCUSS [24] and k-t FOCUSS with sensitivity encoding [20] were used to reconstruct the desired image sequence. The code for k-t FOCUSS [24] was obtained from http://bisp.kaist.ac. kr. Same net reduction factor was used for all methods. For the reconstruction using k-t FOCUSS, images were reconstructed for each coil separately and then combined using square-root of sum-of-squares. The center k-space was fully sampled to estimate the low-resolution image for FOCUSS algorithm, to estimate the sensitivity map for $k$ - $t$ FOCUSS SENSE and the Auto Calibration Data (ACS) for the proposed method. The net reduction factor $R$ is defined as, 

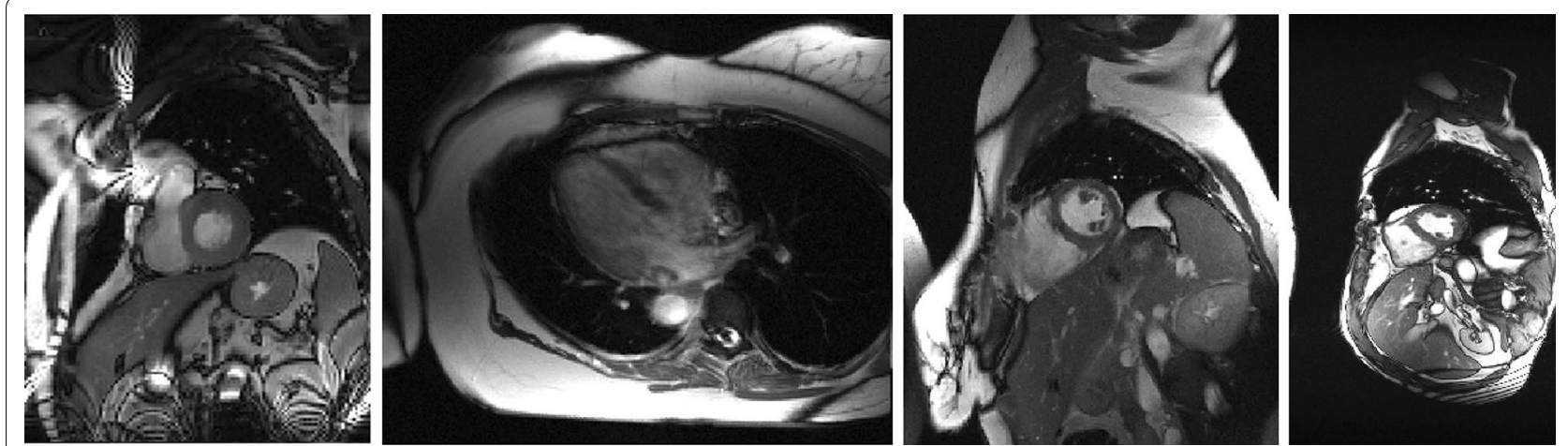

Fig. 2 A representative image frame reconstructed from the fully sampled data for all four datasets
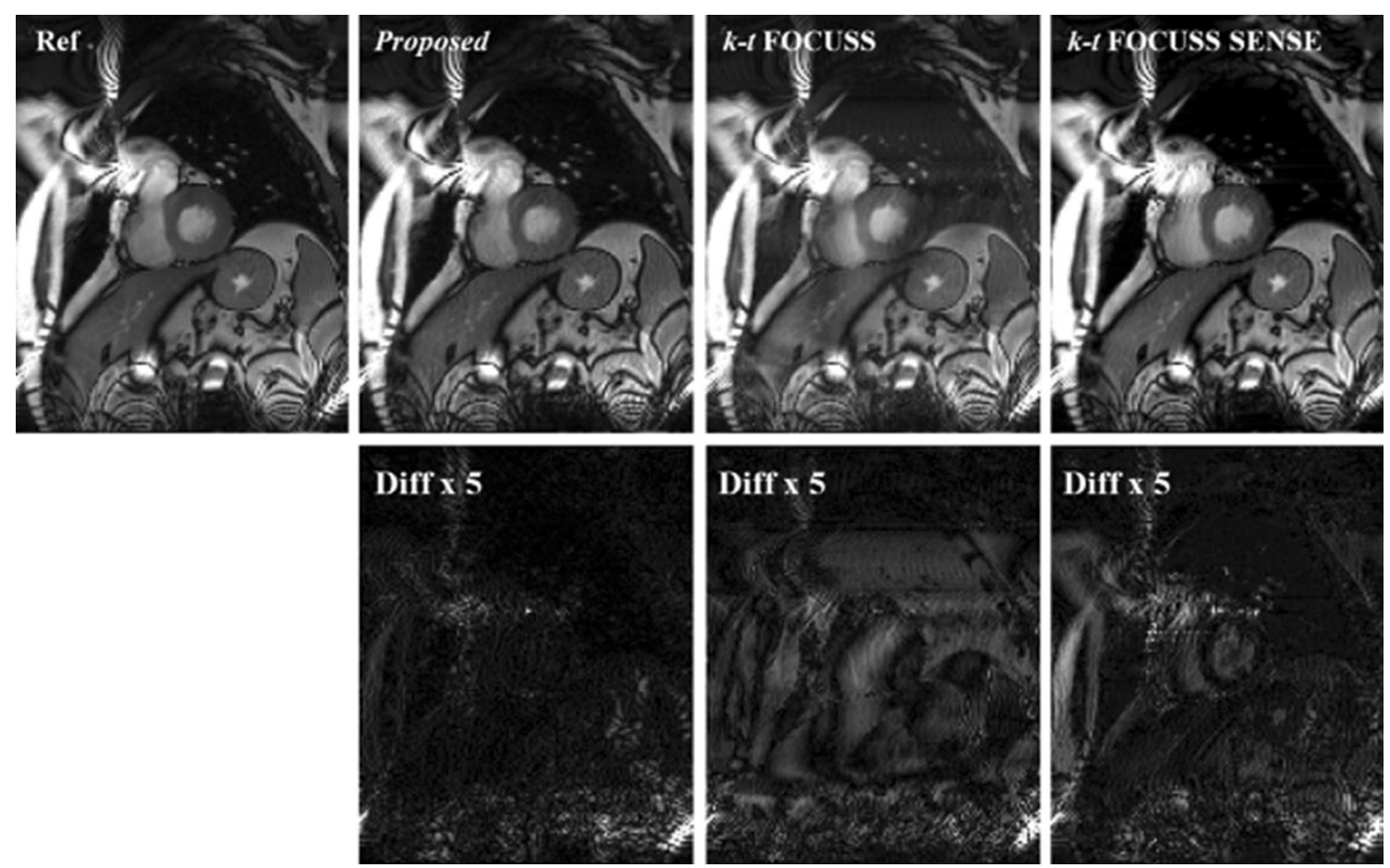

Fig. 3 Reconstructions of the 6th frame of Data 1 using the proposed method (second column), k-t FOCUSS (third column) and k-t FOCUSS SENSE (fourth column). Center 32 phase encoding lines were fully sampled. For the proposed method, the acceleration combination was 3 for CS and 2 for Nonlinear GRAPPA. The net reduction factor was 2.89 for all methods

$$
R=\frac{\text { number of phase encoding lines }}{\frac{\text { number of PE lines }}{R_{1} \times R_{2}}+\text { fully sampled center phase encoding lines }} \text {. }
$$

The images reconstructed from the full k-t data were used as the reference for comparison. All image data presented in Fig. 2 were zero-padded, normalized, and displayed on the same scale, representing the original
FOV. All the computations were carried out on a workstation with Intel i7-3770 $3.40 \mathrm{GHz} \mathrm{CPU}$ and $64 \mathrm{~GB}$ RAM running MATLAB 2019a (Mathworks, Natick, MA). 


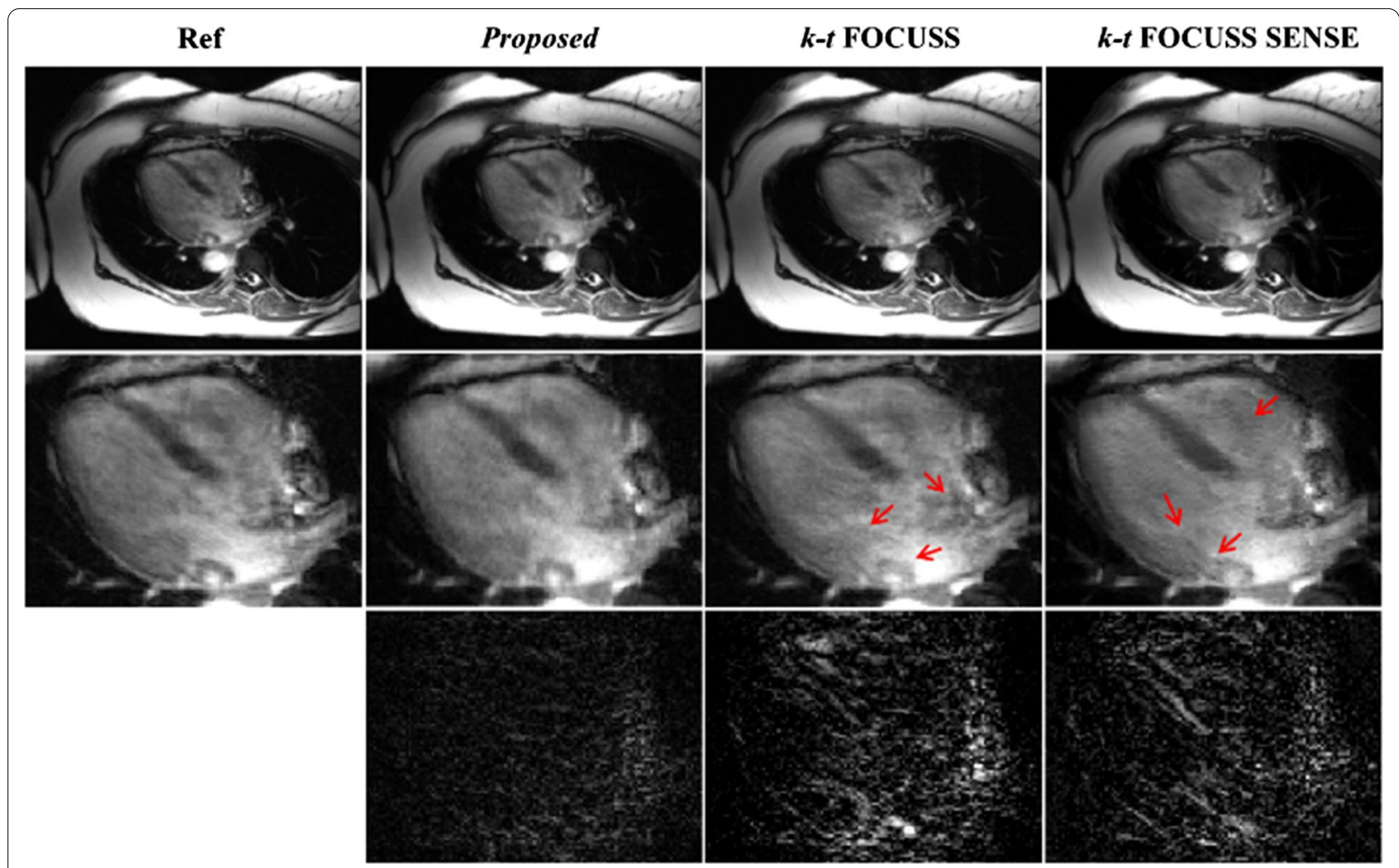

Fig. 4 Reconstructions of the 16th frame of Data 2 using the proposed method (second column), k-t FOCUSS (third column) and k-t FOCUSS SENSE (fourth column). Center 36 phase encoding lines were fully sampled. For the proposed method, the acceleration combination was 4 for CS and 2 for Nonlinear GRAPPA. The net reduction factor was 4.11 for all methods

\section{Results}

Image quality assessment of proposed framework

Figure 3 shows the reconstruction result and the corresponding error images compare with the reference at the sixth frame of the first dataset. The difference images were scaled appropriately to highlight the differences between the reconstructions and the reference images. For the proposed method, the reduction factor was 2 for parallel imaging and 3 for CS. For k-t FOCUSS [24] and k-t FOCUSS SENSE [20], the outer reduction factor was 6 . The center 32 phase encoding lines were fully sampled as the ACS data and estimating the coil sensitivity, which makes the net reduction factor $R=2.89$ for all methods. It is seen in Fig. 3 that the reconstruction using k-t FOCUSS [24] presented aliasing artifacts along the undersampled phase encoding direction. By incorporating sensitivity encoding, the aliasing artifacts were suppressed but presented noise in both cardiac region and background as indicated by arrowheads. The advantage of proposed methods on suppressing aliasing and noise artifacts can be better appreciated in the difference images. Figure 4 shows the reconstruction result of the 16th frame of the second dataset at a net reduction $R=4.11$. For the proposed method, the acceleration combination was 4 for CS and 2 for Nonlinear GRAPPA. Center 36 phase encoding lines were fully sampled. The results of data 2 lead to the same conclusion that the proposed method can suppress more aliasing artifacts and preserve more details than either $\mathrm{k}-\mathrm{t}$ FOCUSS or $\mathrm{k}-\mathrm{t}$ FOCUSS SENSE. It is worth noting that although adding sensitivity encoding could significantly remove the aliasing artifacts, it over-smoothed the image, resulted in a loss of details in the cardiac region. It was mainly due to the inaccurate estimation of the sensitivity map.

To quantitatively evaluate the performance of the proposed method, the normalized mean-square error (NMSE) of the region of interest (ROI) between the reconstruction and the reference were calculated and plotted as a function of time in Fig. 5. The NMSE was calculated by 

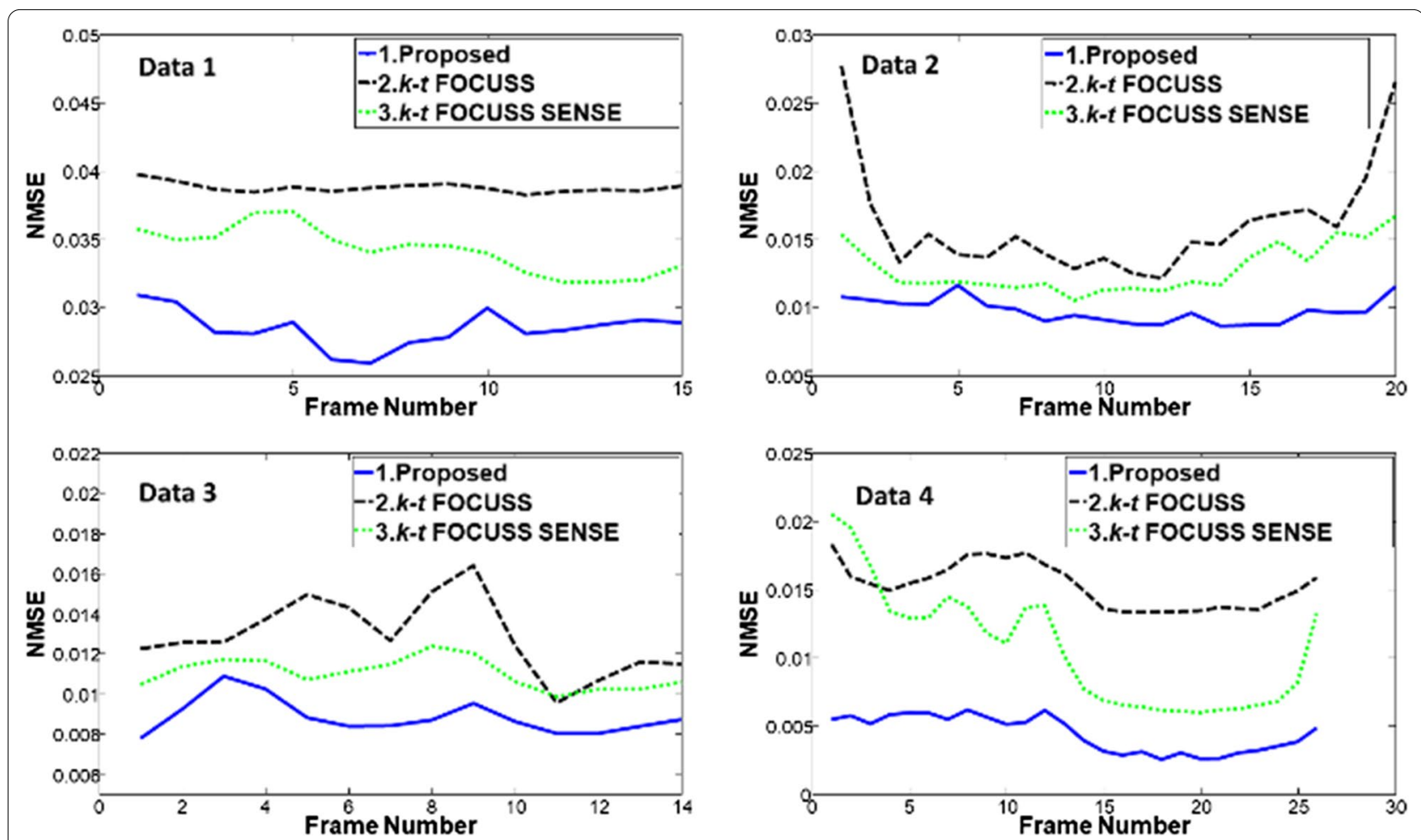

Fig. 5 Frame-by-frame plots of NMSE in the ROI for the proposed method, k-t FOCUSS, and k-t FOCUSS with SENSE with net reduction factor $R=2.89$ for the Data 1, $R=4.11$ for the Data 2, $R=4.14$ for the Data 3, and $R=3.6$ for the Data 4 . The blue solid lines are for the proposed method, green dotted lines for the $k$-t FOCUSS with SENSE, and black dashed lines for the $k$-t FOCUSS

$$
\operatorname{NMSE}\left(\mathbf{I}_{\text {ref }}, \mathbf{I}_{\text {recon }}\right)=\frac{\left\|\mathbf{I}_{\text {ref }}-\mathbf{I}_{\text {recon }}\right\|_{2}^{2}}{\left\|\mathbf{I}_{\text {ref }}\right\|_{2}^{2}} .
$$

It can be seen that the proposed method has a lower NMSE comparing to the other two competing methods at all frames.

To further evaluate the performance of the proposed method in clinical diagnosis, the heart region with the most dynamic motion were considered as the region of interest and zoomed-in for comparison for all methods in the third and fourth dataset. Figure 6 shows the reconstruction in the heart region comparison from different frames of the third dataset at a net reduction factor of 4.14. The acceleration combination of proposed method was 4 for CS and 3 for nonlinear GRAPPA [42-44]. The center 38 phase encoding lines were fully sampled. It can be seen that k-t FOCUSS [24] presented blurring on the image and loses details. By incorporating sensitivity encoding, k-t FOCUSS SENSE improved the blurring but exhibited noise-like artifact. In comparison, the proposed method evidently removed the blurring and the noise. Figure 7 shows the comparison between the proposed method with other three methods with Data 4. It is also suggested that the proposed method is superior at removing motion and aliasing artifact, especially at the cardiac region than the competing methods.

The ability to capture the temporal variation is another important criterion to evaluate the performance of dynamic reconstruction methods. The temporal profiles of the second data with a net reduction factor of $R=4.11$ and Data 4 with a net reduction factor of $R=3.6$ were shown in Fig. 8. It is seen that the k-t FOCUSS method [24] smoothed out the rapid temporal changes, while k-t FOCUSS SENSE [20] suffered from loss of contrast. In comparison, the proposed method preserved most of the temporal variations, especially in regions indicated by the arrowheads.

\section{Choice of acceleration factor combination for CS and PI}

In CS and PI combinations, the error propagating property [28] has been proven to be critical to reconstruction quality; hence, the choice of the acceleration combination is critical to the final result. Figure 9 presented the comparison of the reconstruction quality of Data 3 with different acceleration combinations for CS and PI at the same net reduction factors $R=4(1 \times 4,2 \times 2,4 \times 1)$ and $R=12(2 \times 6,3 \times 4,4 \times 3,6 \times 2)$ with fixed 40 ACS lines. 


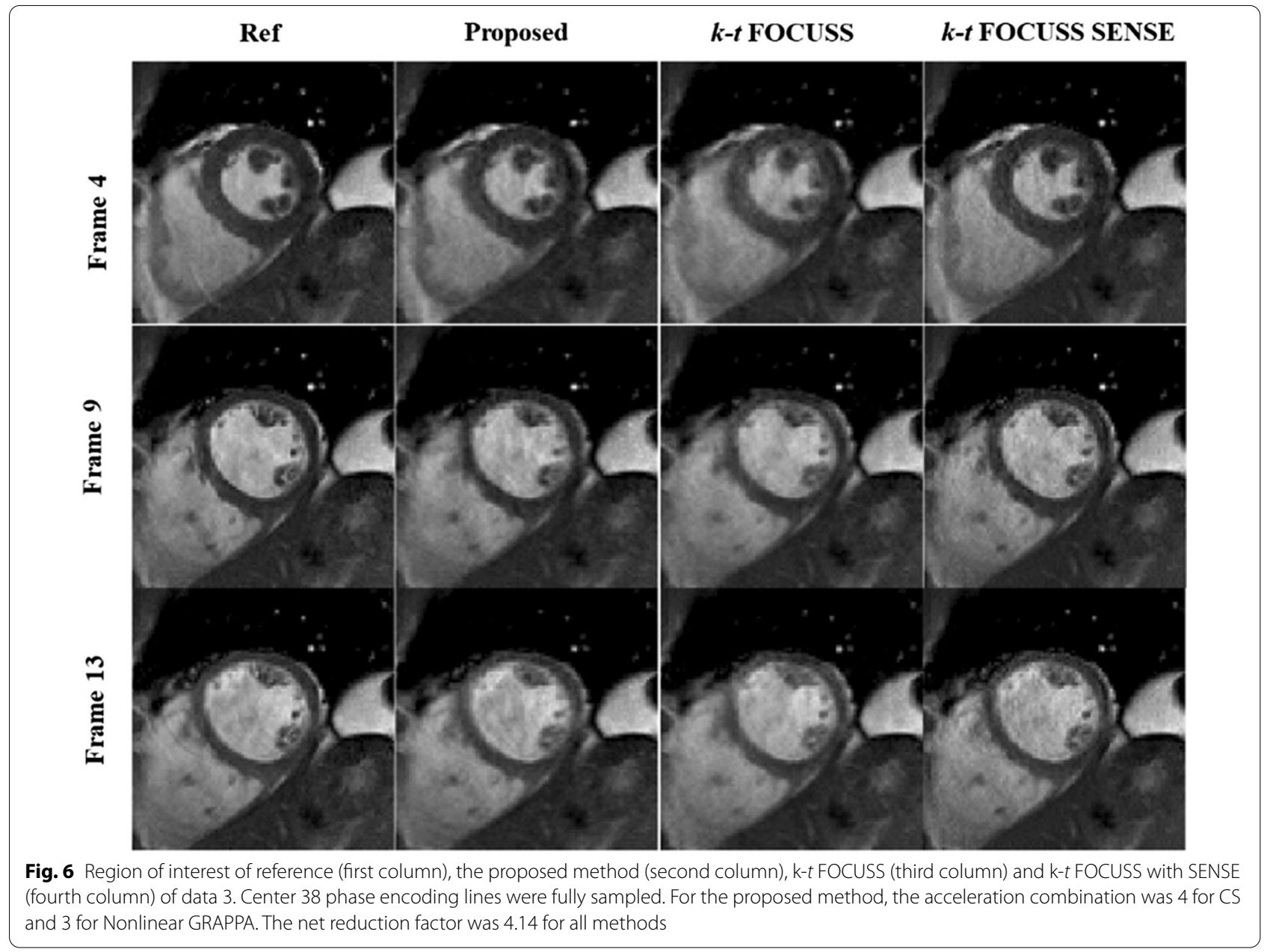

It can be seen that with a fixed number of ACS lines and outer reduction factor (ORF), a small $R_{1}$ will result in lost the de-noised power from CS thus causes large error/ noise amplification, especially at high ORF as shown in $R_{1} \times R_{2}=2 \times 6$; a small $R_{2}$, on the other hand, will result in more severe aliasing artifacts (e.g. $R_{1} \times R_{2}=6 \times 2$ ). In general, if the acceleration factor is evenly distributed, it is best to keep the acceleration at CS step slightly higher than it at PI step in order to avoid error amplification and propagation. This conclusion can be better appreciated at the NMSE plot as shown in Fig. 10.

\section{Effect on number of ACS lines}

It is very important to carefully choose the number of fully sampled ACS lines as it not only affects the performance of nonlinear GRAPPA [33] but also the key factor of the overall net reduction factor. We conducted an experiment to evaluate reconstruction quality with different combinations of $R_{1}, R_{2}$, and ACS lines at a fixed net reduction factor. Figure 11 shows the comparison of the reconstruction quality in terms of NMSE of the third dataset at a fixed net reduction factor of 4.14. It can be seen that the best performance appears when the reduction factor of CS and PI are both low $\left(R_{1} \times R_{2}=3 \times 2, \mathrm{ACS}=28\right)$. However, when the reduction factor is high for PI, even with a large number of ACS, the reconstruction quality was poor as demonstrated in the case of $R_{1} \times R_{2}=3 \times 6, \mathrm{ACS}=48$; If the reduction factor is evenly distributed and the number of ACS lines is fairly large $\left(R_{1} \times R_{2}=4 \times 3\right.$, $\mathrm{ACS}=38 ; R_{1} \times R_{2}=4 \times 4, \mathrm{ACS}=46$ and $R_{1} \times R_{2}=5 \times 4$, $\mathrm{ACS}=50$ ), the reconstruction quality is better than that of the higher acceleration at either CS or PI $\left(R_{1} \times R_{2}=4 \times 6, \mathrm{ACS}=50 ; R_{1} \times R_{2}=8 \times 3, \mathrm{ACS}=52\right)$, although they have more ACS lines. This observation is consistent with that been discussed in the previous section. From what has been discussed above, we can get the conclusion that to get the best reconstruction result, the choice of the number of ACS mainly depends on the acceleration factor on PI, when the accelerations for CS and PI are evenly distributed and keep CS slightly higher than PI if necessary. 

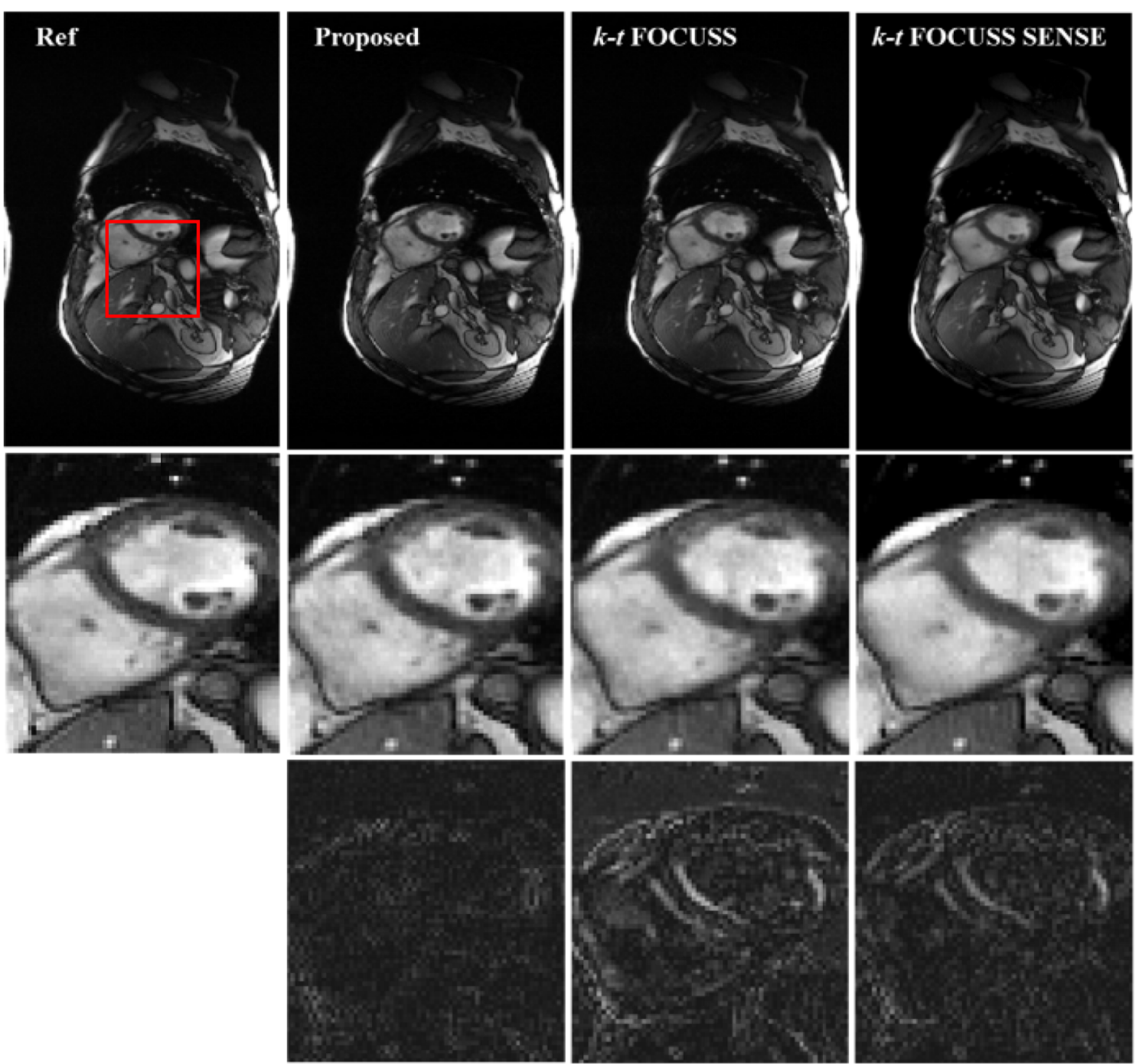

Fig. 7 Reference (first column), the proposed method (second column), k-t FOCUSS (third column) and k-t FOCUSS with SENSE (fourth column) of data 4. Center 32 phase encoding lines were fully sampled. For the proposed method, the acceleration combination was 6 for CS and 2 for Nonlinear GRAPPA. The net reduction factor was 3.6 for all methods

\section{Discussion}

In this paper, we proposed a novel reconstruction framework that effectively combines compressed sensing and parallel imaging for dynamic MRI reconstruction. With a novel sequential reconstruction strategy together and a tailored sampling scheme, the proposed method was shown to be able to better suppress aliasing artifacts and noise at high accelerations, comparing to the conventional compressed sensing method that incorporated with sensitivity encoding. Although the FOCUSS and nonlinear GRAPPA techniques used in the presented study was previously proposed, the novelty of this study is not to focus on proposing a single reconstruction algorithm, but rather to develop a reconstruction flowchart that can better combine compressed sensing and parallel imaging to ensure the capability to recover high-quality images from maximized acceleration. The reconstruction strategies for each step could easily be replaced by more advanced techniques, i.e. low-rank model [31, 32], dictionary learning $[33,34]$ for CS reconstruction; E-SPiRiT for the parallel imaging reconstruction [35].

There are many researches that attempt to combine CS and PI [20, 25, 28]. In [28], it used a very similar framework but was limited to the static images. When applying to dynamic MRI, taking the temporal information into account could further reduce the amount of data required for reconstruction. In addition, the 


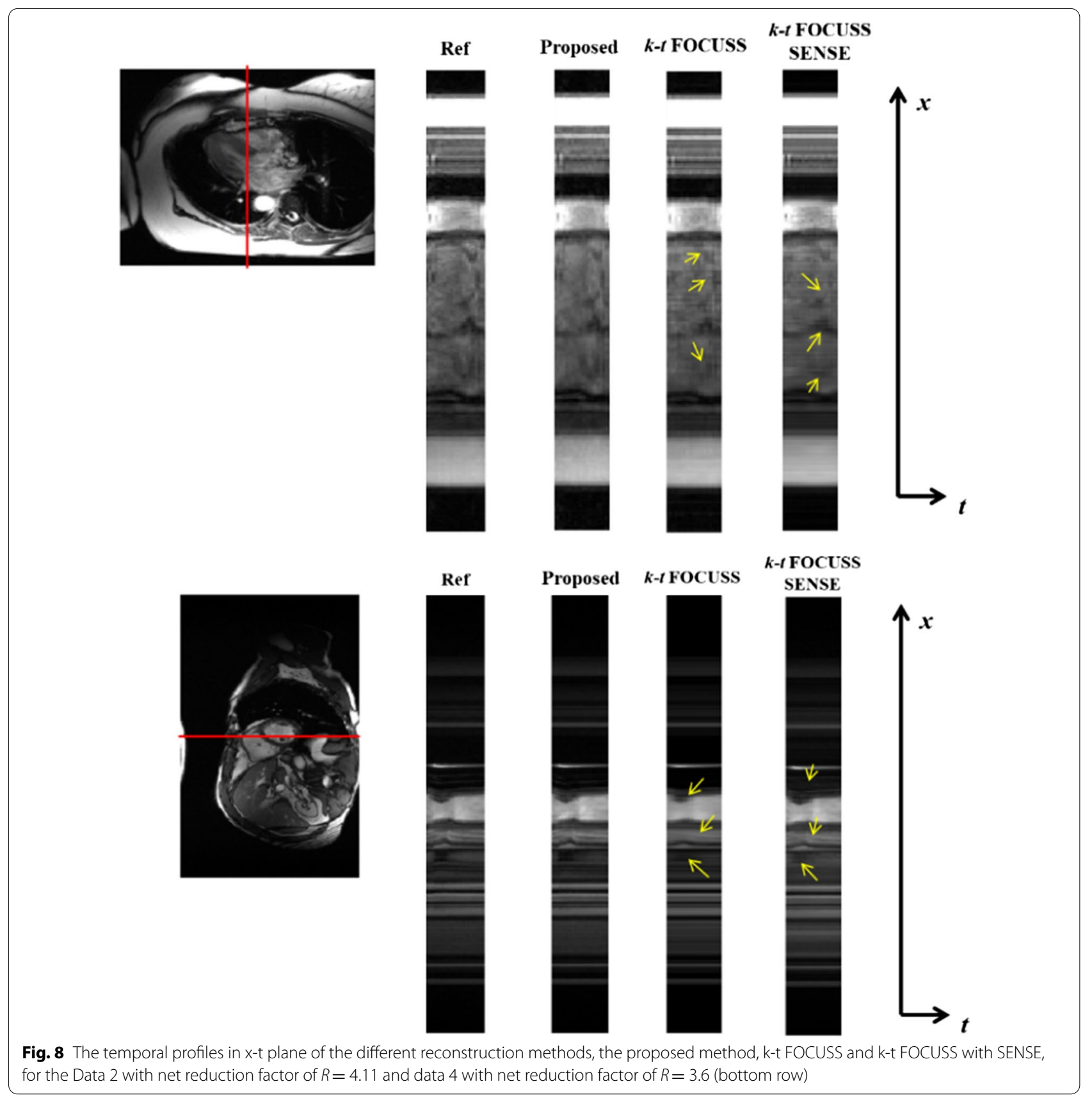

inaccurate estimation of the sensitivity information may futher degrade the image quality. In the methods mentioned in Refs. [20] and [25], the sensitivity encoding is incorporated into the Fourier encoding in the data consistency term. Such algorithm exploits the joint sparsity information from all coils, representing the distributed compressed sensing framework. However, such a simple combination is difficult to maximize the accelerations that can be achieved by each individual method because each method has different sampling requirements. In contrary, the proposed framework decouples the acceleration into two sequential steps to maximize the advantages gain from both PI and CS. Experimental results confirmed that the proposed framework can better capture the low contrast cardiac blood flow and preserve more temporal information than the conventional methods. Additionally, employing nonlinear GRAPPA instead of SENSE may also contribute to better suppress noise and remove artifacts. Jung et al. [45] compared the performance of $k$-t SENSE 

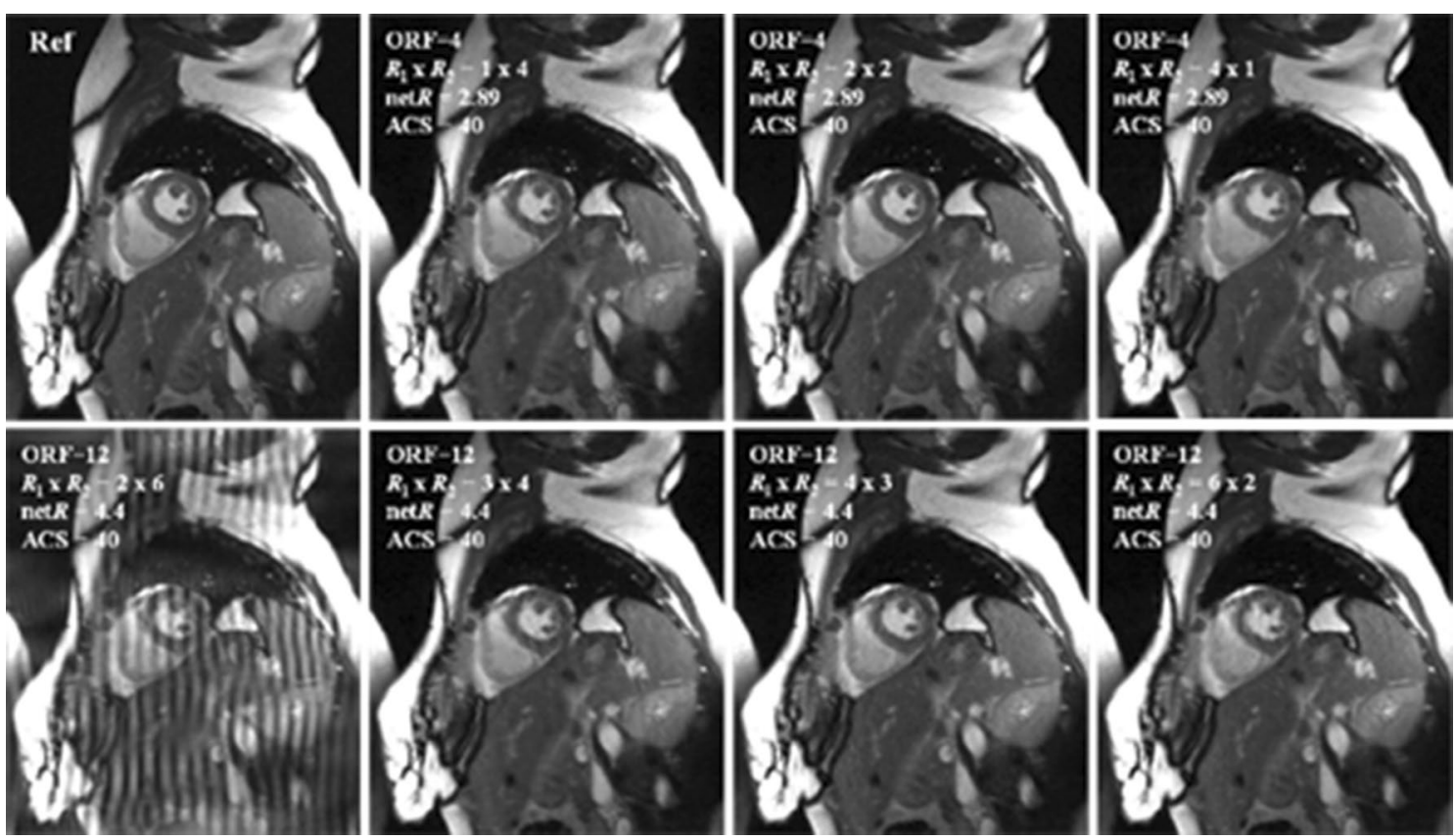

Fig. 9 The proposed method of the Data 3 with different combinations of reduction factors
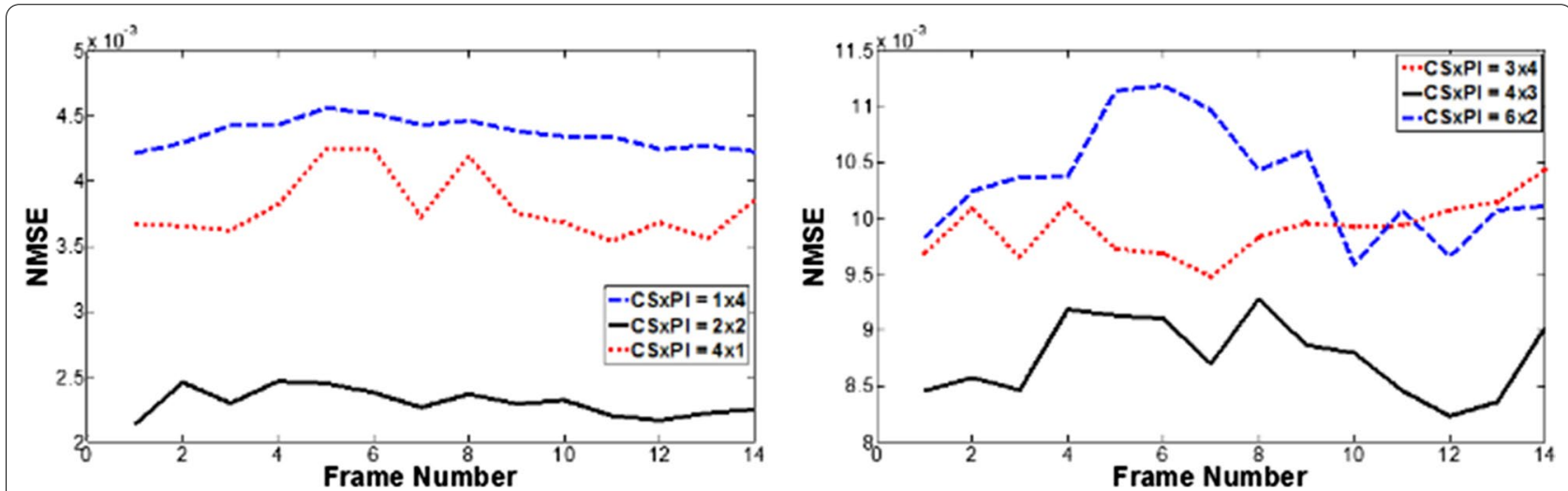

Fig. 10 The NMSE plots of the proposed method reconstructions of the Data 3 with different combinations of reduction factors

and k-t GRAPPA applied to cardiac cine and phasecontrast images. They observed that although both methods provide excellent image quality and temporal fidelity for different acceleration factors, k-t GRAPPA demonstrated less spatially varying noise than $k-t$ SENSE. In addition, the sampling trajectory demonstrated in this study uses the combination of random sampling patterns over a uniform undersampling pattern in a Cartesian manner. However, the proposed method could also be applied to non-Cartesian cases, i.e. radial or spiral subsampling, so that many advanced techniques [35-38] could be applied to the proposed framework to achieve better performance.
The proposed method was demonstrated using cardiac cine imaging in this study. However, as it was designed to maximize the acceleration of the data acquisition and improve spatial.-temporal resolution, the proposed method is also expected to benefit other dynamic MR applications, such as dynamic contrast-enhanced MRI and dynamic MR angiography [21, 23]. Nevertheless, it is imperative to choose the best reconstruction strategy for each step based on the specific characteristics of the signal within the application. For instance, in dynamic images whose signal is not periodic, the Fourier transform might not be the best choice to sparsifying the images. In 


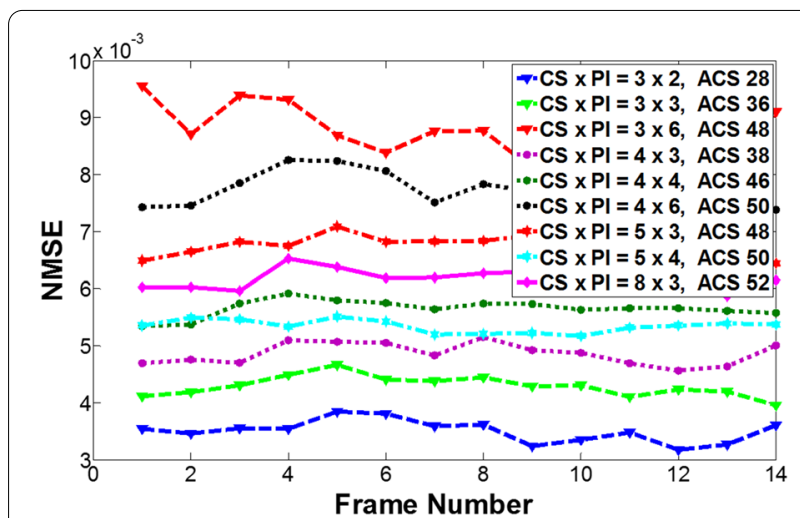

Fig. 11 The quality comparison of the proposed method in terms of NMSE of the Data 3 at a fixed net reduction factor of 4.14 with the different combinations of $R_{1}, R_{2}$ and ACS lines

the CS step, Karhunen-Loeve transform or principal component analysis might have better capability to sparse the image thus provides a more accurate k-space reconstruction for the PI step.

While deep-learning techniques for accelerating MR acquisition have gained significant attention in recent years, parallel imaging and compressed sensing continue to play important roles in both clinical practices and research due to their robustness, reproducibility, and repeatability, which have been validated by clinical studies. Although deep learning holds great potential, few studies have evaluated its reliability in certain clinical applications. In light of this, the proposed method still holds great promise for its ability to rapidly adapt to current clinical applications.

The proposed method has higher computational complexity than the conventional compressed sensing based methods with sensitivity encoding. Particularly, in the CS step of the proposed method, the FOCUSS algorithm approximates the solution to the $\ell_{1}$ minimization through iteratively reweighted $\ell_{2}$ minimization. The computational complexity is exactly equivalent to that in k-t FOCUSS [24]; In the PI step, the nonlinear GRAPPA [42] is about 2-5 times that of the conventional GRAPPA [13]. So overall the proposed method has a higher computational complexity than that of $\mathrm{k}-\mathrm{t}$ FOCUSS [24] or k-t FOCUSS with SENSE [20] due to the nonlinear GRAPPA process. In our current implementation, the total computation time is about $323 \mathrm{~s}$ of the proposed method comparing with $19 \mathrm{~s}$ that of $\mathrm{k}-\mathrm{t}$ FOCUSS [24] and $33 \mathrm{~s}$ that of $\mathrm{k}-\mathrm{t}$ FOCUSS with SENSE [20]. In addition to this, this study has limitations. First, the reconstruction techniques used in this study, i.e. $\mathrm{k}-\mathrm{t}$
FOCUSS and nonlinear GRAPPA, were a bit out of date. Some more advanced or state-of-the-art methods could be applied. This is warranted in future studies. Second, the proposed framework was only demonstrated on a limited number of datasets. A more thorough validation of the proposed framework should be conducted with clinical and patient data before its translation into clinical practice is possible-although the current datasets are adequate as the proof-of-concept for the proposed method.

\section{Conclusion}

We proposed a novel joint framework to sufficiently combines compressed sensing technique with parallel imaging to accelerate dynamic MRI. The proposed method decouples the reconstruction process into two sequential steps: use the CS method to reconstruct a series of aliased dynamic images from the highly undersampled $\mathrm{k}$-space data, and use the nonlinear GRAPPA method to missing $\mathrm{k}$-space data for the original image. The in vivo experiments of cardiac cine imaging suggested that the proposed method can preserve more spatial details and temporal variations of dynamic cardiac images than the state-of-the-art dynamic imaging methods such as the classical k-t FOCUSS method either with or without sensitivity information.

\section{Acknowledgements}

We thank Dr. Jong Chul Ye for making the k-t FOCUSS code available online. We also would like to thank Dr. E. V. R. Dibella for providing cardiac cine datasets.

\section{Authors' contributions}

$\mathrm{ZH}$ and $\mathrm{CZ}$ conducted the experiments, performed the computations and drafted the main manuscript text. XZ, LK and JY summarized the results and prepared the figures; XW and JL verified the analytical methods and helped to revise the manuscript. YZ conceived of the presented idea, supervised the findings of this work and revised the manuscript. All authors discussed the results and contributed to the final manuscript. All authors read and approved the final manuscript.

\section{Funding}

This work was supported in part by the Sanming Project of Medicine in Shenzhen (SZSM201812005), and the Joint Special Fund for Local Universities in Yunnan Science and Technology Department (2019FH001-070).

\section{Availability of data and materials}

The datasets used and/or analyzed during the current study available from the corresponding author upon request.

\section{Declarations}

\section{Ethics approval and consent to participate}

This study was approved by the Shenzhen children's hospital. Written informed consent was obtained from all volunteers in accordance with the 
institutional review board policy and with the 1964 Helsinki declaration and its later amendments or comparable ethical standards.

\section{Consent for publication}

Not applicable.

\section{Competing interests}

The authors declare that they have no competing interests.

\section{Author details}

'Shenzhen Children's Hospital, Shenzhen 518038, Guangdong, China. ${ }^{2}$ Shenzhen College of Advanced Technology, University of Chinese Academy of Sciences, Shenzhen 518055, Guangdong, China. ${ }^{3}$ School of Physics and Electronic Engineering, Yuxi Normal University, Yuxi 653100, Yunnan, China. ${ }^{4}$ Hong Kong Sanatorium and Hospital, 5 A Kung Ngam Village Road, Shau Kei Wan, Hong Kong, China.

Received: 19 July 2021 Accepted: 6 October 2021 Published online: 01 December 2021

\section{References}

1. Liang Z-P, Lauterbur PC. An efficient method for dynamic magnetic resonance imaging. IEEE Trans Med Imaging. 1994;13(4):677-86.

2. Van Vaals JJ, Brummer ME, Thomas Dixon W, Tuithof HH, Engels H, Nelson RC, Gerety BM, Chezmar JL, Den Boer JA. "Keyhole" method for accelerating imaging of contrast agent uptake. J Magn Reson Imaging. 1993;3(4):671-5.

3. Riederer SJ, Tasciyan T, Farzaneh F, Lee JN, Wright RC, Herfkens RJ. MR fluoroscopy: technical feasibility. Magn Reson Med. 1988;8(1):1-15.

4. Madore B, Glover GH, Pelc NJ. Unaliasing by Fourier-encoding the overlaps using the temporal dimension (UNFOLD), applied to cardiac imaging and fMRI. Magn Reson Med. 1999;42(5):813-28.

5. Liang Z-P. Spatiotemporal imagingwith partially separable functions. In: 2007 4th IEEE international symposium on biomedical imaging: from nano to macro. IEEE; 2007. p. 988-91

6. Brinegar C, Zhang H, Wu Y-JL, Foley LM, Hitchens TK, Ye Q, Pocci D, Lam F, Ho C, Liang Z-P. Real-time cardiac MRI using prior spatial-spectral information. In: 2009 annual international conference of the IEEE engineering in medicine and biology society. IEEE; 2009. p. 4383-86

7. L Liang ZP, Jiang H, Hess CP, Lauterbur PC. Dynamic imaging by model estimation. Int J Imaging Syst Technol. 1997;8(6):551-7.

8. Sumbul U, Santos JM, Pauly JM. Improved time series reconstruction for dynamic magnetic resonance imaging. IEEE Trans Med Imaging. 2009;28(7):1093-104.

9. Aggarwal N, Bresler Y. Patient-adapted reconstruction and acquisition dynamic imaging method (PARADIGM) for MRI. Inverse Problems. 2008;24(4):045015.

10. Tsao J, Boesiger P, Pruessmann KP. k-t BLAST and k-t SENSE: dynamic MRI with high frame rate exploiting spatiotemporal correlations. Magn Reson Med. 2003;50(5):1031-42.

11. Sodickson DK, Manning WJ. Simultaneous acquisition of spatial harmonics (SMASH): fast imaging with radiofrequency coil arrays. Magn Reson Med. 1997;38(4):591-603.

12. Pruessmann KP, Weiger M, Scheidegger MB, Boesiger P. SENSE: sensitivity encoding for fast MRI. Magn Reson Med. 1999;42(5):952-62.

13. Griswold MA, Jakob PM, Heidemann RM, Nittka M, Jellus V, Wang J, Kiefer B, Haase A. Generalized autocalibrating partially parallel acquisitions (GRAPPA). Magn Reson Med. 2002;47(6):1202-10.

14. Ying L, Liang Z-P. Parallel MRI using phased array coils. IEEE Signal Process Mag. 2010;27(4):90-8.

15. Candès EJ, Romberg J, Tao T. Robust uncertainty principles: exact signal reconstruction from highly incomplete frequency information. IEEE Trans Inf Theory. 2006;52(2):489-509.

16. Donoho DL. Compressed sensing. IEEE Trans Inf Theory. 2006;52(4):1289-306.
17. Lustig M, Donoho D, Pauly JM. Sparse MRI: the application of compressed sensing for rapid MR imaging. Magn Reson Med. 2007;58(6):1182-95.

18. Lustig M, Santos JM, Donoho DL, Pauly JM. kt SPARSE: High frame rate dynamic MRI exploiting spatio-temporal sparsity. In: Proceedings of the 13th annual meeting of ISMRM. Seattle; 2006.

19. Jung H, Ye JC, Kim EY. Improved k-t BLAST and k-t SENSE using FOCUSS. Phys Med Biol. 2007;52(11):3201.

20. Gamper U, Boesiger P, Kozerke S. Compressed sensing in dynamic MRI. Magn Reson Med. 2008;59(2):365-73.

21. Bilgin A, Trouard T, Altbach M, Raghunand N. Three dimensional compressed sensing for dynamic MRI. In: Proceedings of the 16th ISMRM scientific meeting and exhibition. 2008.

22. Adluru G, McGann C, Speier P, Kholmovski EG, Shaaban A, DiBella EV. Acquisition and reconstruction of undersampled radial data for myocardial perfusion magnetic resonance imaging. J Magn Reson Imaging. 2009;29(2):466-73.

23. Chen L, Schabel MC, DiBella EV. Reconstruction of dynamic contrast enhanced magnetic resonance imaging of the breast with temporal constraints. Magn Reson Imaging. 2010;28(5):637-45.

24. Jung H, Sung K, Nayak KS, Kim EY, Ye JC. k-t FOCUSS: a general compressed sensing framework for high resolution dynamic MRI. Magn Reson Med. 2009:61(1):103-16.

25. Otazo R, Kim D, Axel L, Sodickson DK. Combination of compressed sensing and parallel imaging for highly accelerated first-pass cardiac perfusion MRI. Magn Reson Med. 2010;64(3):767-76.

26. Liang D, DiBella EV, Chen RR, Ying L. k-t ISD: dynamic cardiac MR imaging using compressed sensing with iterative support detection. Magn Reson Med. 2012;68(1):41-53.

27. Liu B, Zou YM, Ying L: SparseSENSE: application of compressed sensing in parallel MRI. In: 2008 International conference on information technology and applications in biomedicine. IEEE; 2008. p. 127-30.

28. Liang D, Liu B, Wang J, Ying L. Accelerating SENSE using compressed sensing. Magn Reson Med. 2009;62(6):1574-84.

29. Lustig M, Pauly JM. SPIRiT: iterative self-consistent parallel imaging reconstruction from arbitrary k-space. Magn Reson Med. 2010;64(2):457-71.

30. Murphy M, Alley M, Demmel J, Keutzer K, Vasanawala S, Lustig M. Fast \$\} ell_1 \$-SPIRiT compressed sensing parallel imaging MRI: scalable parallel implementation and clinically feasible runtime. IEEE Trans Med Imaging. 2012;31(6):1250-62.

31. Zhao B, Haldar JP, Brinegar C, Liang Z-P: Low rank matrix recovery for realtime cardiac MRI. In: 2010 IEEE international symposium on biomedical imaging: from nano to macro. IEEE; 2010. p. 996-99.

32. Zhao B, Lu W, Hitchens TK, Lam F, Ho C, Liang ZP. Accelerated MR parameter mapping with low-rank and sparsity constraints. Magn Reson Med. 2015;74(2):489-98.

33. Caballero J, Rueckert D, Hajnal JV: Dictionary learning and time sparsity in dynamic MRI. In: International conference on medical image computing and computer-assisted intervention. Springer; 2012. p. 256-63.

34. Huang Y, Paisley J, Lin Q, Ding X, Fu X, Zhang X-P. Bayesian nonparametric dictionary learning for compressed sensing MRI. IEEE Trans Image Process. 2014:23(12):5007-19.

35. Uecker M, Lai P, Murphy MJ, Virtue P, Elad M, Pauly JM, Vasanawala SS, Lustig M. ESPIRiT-an eigenvalue approach to autocalibrating parallel MRI: where SENSE meets GRAPPA. Magn Reson Med. 2014;71(3):990-1001.

36. Feng L, Grimm R, Block KT, Chandarana H, Kim S, Xu J, Axel L, Sodickson DK, Otazo R. Golden-angle radial sparse parallel MRI: combination of compressed sensing, parallel imaging, and golden-angle radial sampling for fast and flexible dynamic volumetric MRI. Magn Reson Med. 2014;72(3):707-17.

37. Bustin A, Fuin N, Botnar RM, Prieto C. From compressed-sensing to artificial intelligence-based cardiac MRI reconstruction. Front Cardiovasc Med. 2020;7:17.

38. Ke Z, Cheng J, Ying L, Zheng H, Zhu Y, Liang D. An unsupervised deep learning method for multi-coil cine MRI. Phys Med Biol. 2020;65(23):235041. 
39. Sandino CM, Lai P, Vasanawala SS, Cheng JY. Accelerating cardiac cine MRI using a deep learning-based ESPIRiT reconstruction. Magn Reson Med. 2021;85(1):152-67.

40. Zhou Y, Chang Y, Liang D, Ying L. kt CSPI: a dynamic MRI reconstruction framework for combining compressed sensing and parallel imaging. In: 2012 9th IEEE international symposium on biomedical imaging (ISBI). IEEE; 2012. p. 410-13.

41. Zhou Y, Chang Y, Ying L: kt CS-NLG: Dynamic imaging reconstruction with compressed sensing and nonlinear GRAPPA. In: Proceedings of the 20th annual meeting of the international society for magnetic resonance in medicine. 2012; p. 1263

42. Chang Y, Liang D, Ying L. Nonlinear GRAPPA: a kernel approach to parallel MRI reconstruction. Magn Reson Med. 2012;68(3):730-40.

43. Wang H, Chang Y, Ying L, Liu X, Liang D: Nonlinear GRAPPA Reconstruction with Virtual Coil Conception. In: Proceedings of the joint annual meeting ISMRM-ESMRMB 2018. 2018.

44. Wang H, Jia S, Chang Y, Zhu Y, Zou C, Li Y, Liu X, Zheng H, Liang D. Improving GRAPPA reconstruction using joint nonlinear kernel mapped and phase conjugated virtual coils. Phys Med Biol. 2019;64(14):14NT01.

45. Jung B, Kozerke S: Comparison of kt-SENSE and kt-GRAPPA applied to cardiac cine and phase contrast imaging. In: Proceedings of the 17th annual meeting of ISMRM. 2009.

\section{Publisher's Note}

Springer Nature remains neutral with regard to jurisdictional claims in published maps and institutional affiliations.

- fast, convenient online submission

- thorough peer review by experienced researchers in your field

- rapid publication on acceptance

- support for research data, including large and complex data types

- gold Open Access which fosters wider collaboration and increased citations

- maximum visibility for your research: over $100 \mathrm{M}$ website views per year

At BMC, research is always in progress.

Learn more biomedcentral.com/submissions 